\title{
Integrative Health in the Veterans Health Administration
}

\author{
Benjamin Kligler, MD, MPH
}

$\mathbf{O}$ VER THE PAST 5 YEARS, the Veterans Health Administration (VHA) has made significant progress in expanding the availability of complementary and integrative health $(\mathrm{CIH})$ services to veterans, with a focus on the use of these approaches for treatment of pain and mental health conditions. $\mathrm{CIH}$ is seen as an essential component of a larger VHA vision based on the Whole Health model—delivering healthcare that centers on the goals and needs of each veteran. The core concept of the Whole Health model is that, instead of starting with the veteran's problem, the VHA must approach the veteran as a whole person. Rather than focusing on "what is the matter" with the veteran, Whole Health focuses on "what matters to the veteran." With that as its focus, the VHA is redesigning its medical delivery model around the whole person rather than a particular disease or medical problem.

The Whole Health model-which we hope will eventually be available to every veteran at every VHA facilityconsists of three core components, revolving around the idea of a personal health plan in which what matters to the veteran is the central organizing concept:

1. The Pathway-Whole Health Peer Partners work with veterans in either group or individual settings, exploring their missions, purposes, and aspirations"what matters to them"-and beginning to develop their overarching personal health plans.

2. Well-Being Programs-The model provides programs that include skill building and support, CIH services, health coaching, and personal health planning. Programs are designed for the individual (not based just on the diagnosis or disease) and include proactive, integrative health approaches, such as stress reduction, yoga, T'ai Chi, mindfulness, nutrition, and acupuncture. These programs might be based at the facility or, in some cases, at a veteran's center, or in the local community.

3. Whole Health Clinical Care-Our goal is to train clinicians who are delivering care to veterans in how to organize that care around the personal health plan and to place an emphasis on self-management support and shared decision-making. An extensive training effort aimed at VHA clinicians has been underway for several years to help move this transformation forward.

Many sites around the VHA are already offering $\mathrm{CIH}$ services - this included $93 \%$ of facilities as of $2015 .{ }^{1}$ Yet, the number of veterans receiving these services is still small and the availability of these services remains limited. A major initiative is now underway to expand access to $\mathrm{CIH}$ nationally, with a primary focus on a number of $\mathrm{CIH}$ therapies with the best evidence of effectiveness, including acupuncture, chiropractic, T'ai Chi, yoga, and meditation. The short-term goal is for every facility to offer at least two $\mathrm{CIH}$ approaches for veterans with pain conditions. Obviously, the widespread awareness of the opioid epidemic, and the VHA's success over the past 3 years in curtailing opioid prescribing, is helping create momentum for this expanded access to $\mathrm{CIH}$.

The Integrative Health Coordinating Center (IHCC), which operates within the Office of Patient Centered Care \& Cultural Transformation, was established in 2014 and charged with developing and implementing $\mathrm{CIH}$ strategies in clinical activities, education, and research. The IHCC is responsible for identifying, standardizing, and operationalizing integrative health therapies for veterans. Its primary roles are identifying and removing VHA barriers to $\mathrm{CIH}$ services, serving as the source of information for $\mathrm{CIH}$ clinical practices and education, and defining and driving measures for assessing the effectiveness of CIH approaches. Some of the current critical priorities at the IHCC include the effort to formalize the inclusion of $\mathrm{CIH}$ approaches in the medical benefits package; the development of new occupations, qualification standards, and position descriptions for $\mathrm{CIH}$ professionals (including licensed acupuncturists and massage therapists, yoga, T'ai Chi, and meditation

Integrative Health Coordinating Center, Veterans Health Administration, Washington, DC.

The views expressed are those of the author and do not reflect the official policy or position of the Uniformed Services University of the Health Sciences, the Department of Defense or the United States Air Force. 
instructors) to facilitate hiring and quality care; and the effort to develop infrastructure and coding practices for tracking utilization of $\mathrm{CIH}$ approaches within the VHA.

A major development in the past year was the Comprehensive Addiction and Recovery Act (CARA), new legislation passed by Congress in July of 2016, which mandates a significant expansion of $\mathrm{CIH}$ services across the VHA as well as increased attention to education and research in $\mathrm{CIH}$ within the VHA. Specifically, the CARA law requires the establishment of 153 -year pilots around the country to examine the process and the effect of expanding CIH services. Although the law focuses largely on pain and mental health, these pilot sites will also be free to offer $\mathrm{CIH}$ in support of other chronic disease conditions and in support of veterans' well-being as well.

As part of the VHA's ongoing commitment to the Whole Health model, and in response to this mandate in the CARA legislation, in October 2016 the Veterans Integrated Service Network (VISN) directors agreed unanimously to establish one Whole Health flagship demonstration site within each of the 18 VISNs starting in fiscal year 2018 (FY18). Given that $\mathrm{CIH}$ is considered a core component of the Whole Health model, these flagship sites will provide an unprecedented opportunity to refine the process of CIH delivery to veterans and to study its effect. A detailed planning process is now underway to prepare for the selection and rollout of these 18 demonstration sites.

Acupuncture in particular has gained a great deal of traction within the VA system, and is in great demand as part of the response to the opioid epidemic and to the need for effective pain treatment. Many VHA centers are delivering acupuncture in group settings, which allows for more patients to be treated with the limited resources available. The recently completed partnership among the IHCC, the VA Office of Pain Management, and the Defense \& Veterans Center for Integrative Pain Management-a Joint Incentive Fund (JIF)-supported project called the Acupuncture Training Across Clinical Settings (ATACS) program-has led to a significant expansion of the availability of acupuncture to veterans. This program supported medical acupuncture training for 88 providers, and also rolled out large-scale training in Battlefield Acupuncture (BFA). BFA is a simple 5point auricular technique used for treating acute and chronic pain. To date, more than 1000 providers have been trained in BFA through the JIF ATACS program, and we have more than 50 BFA VHA instructors trained as well. With the completion of the JIF, the IHCC is now supporting continuing training and expansion of BFA, which has met with extremely positive feedback from veterans.

We have also been working for several years to be able to hire licensed acupuncturists within the VHA system. Because this was not an occupation formally recognized by the federal government, up to this point VHA facilities have only been able to hire LAcs as contractors, rather than as full employees. As a consequence, most of the acupuncture being delivered in the VHA is performed by physicianacupuncturists - not necessarily the most cost-effective way to deliver acupuncture on a large scale to veterans. We expect that, within the next year, the long process of "creating" this new occupation for federal hiring will be completed, and we will be able to expand the workforce delivering acupuncture in the VHA.

Another major issue regarding acupuncture in the VHAas it is in the private sector as well-is the lack of clear guidelines for how many acupuncture treatments are needed for effective treatment of specific conditions. The current published literature does not provide clear guidance on this-although a range of 8-12 treatments seems to be commonly recommended. A work group within the VHA was recently launched to develop clearer guidelines on this issue. This is particularly important given that a significant amount of VHA care is now being delivered outside the walls of the facilities by community practitioners-and there needs to be a clear standard for what the VHA will support in terms of acupuncture delivered in the community as well.

Finally, a major priority for us is the development of rigorous outcome measurements regarding the effectiveness of all categories of $\mathrm{CIH}$ in the veteran population. The planning for the 18 flagship sites includes a comprehensive outcome measurement strategy looking at measures of pain, mental health, and quality of life as well as of veteran satisfaction, cost, and utilization. We are currently launching with colleagues from VHA Health Services Research \& Development a study specifically focused on implementation and effectiveness of BFA. Ultimately, the full integration of acupuncture and other $\mathrm{CIH}$ services into VHA care will depend on successfully measuring and documenting the outcomes of that care on what matters to the veterans over the course of this coming expansion phase.

\section{REFERENCE}

1. Healthcare Analysis \& Information Group (HAIG). A Field Unit of the Office of Strategic Planning \& Analysis within the Office of the ADUSH for Policy and Planning. Page 9. Online document at: http://scienceblogs.com/insolence/files/2016/07/ FY2015_VHA_CIH_signedReport.pdf Accessed March 1, 2017.

Address correspondence to:

Benjamin Kligler, $M D, M P H$

Integrative Health Coordinating Center Veterans Health Administration 810 Vermont Avenue $\mathrm{NW}$ Washington, DC 20420

E-mail: benjamin.kligler@va.gov 